\title{
Real-Time Polarimetric Optical High-Voltage Sensor Using Phase-Controlled Demodulation
}

\author{
Fernando da Cruz Pereira ${ }^{\circledR}$, José Henrique Galeti, Member, IEEE, Ricardo Tokio Higuti, Member, IEEE, \\ Michael J. Connelly ${ }^{\mathbb{D}}$, Senior Member, IEEE, and Cláudio Kitano (1)
}

\begin{abstract}
Modern smart grid designs demand reliable sensors throughout the power delivery systems, not only to monitor the quality of the electrical energy generated by different technologies (wind, solar, hydroelectric, thermoelectric, nuclear), but also as an integral part of the generation, transmission, and distribution networks control systems. Laser polarimetric interferometry is a very suitable technique for the implementation of high sensitivity measurement instruments for various physical quantities, in particular, for high-voltage sensing. However, because the polarimetric technique is extremely sensitive to small refractive indexes variations induced in electro-optic materials by the electric field or voltage to be measured, it is also sensitive to spurious environmental quantities such as temperature variations. In this work, a novel optical voltage sensor (OVS) in conjunction with an efficient real-time method for optical phase demodulation and a PI feedback control method for signal fading suppression is demonstrated. Linearity for sinusoidal voltages having amplitudes up to $8.2 \mathrm{kV}$ was evaluated, with an accuracy of $0.2 \%$ in the OVS nominal operating range. The OVS bandwidth of $5 \mathrm{kHz}$ is sufficient for most electrical power quality sensor applications. Nonsinusoidal signals with high harmonic content were tested resulting in a total harmonic distortion that differed by only $0.12 \%$ from that measured by an HV reference probe. Responses to transient disturbances were also determined, showing the capability of the system to detect spikes.
\end{abstract}

Index Terms-High-voltage sensor, instrumentation, interferometry, measurement, metrology, phase measurement.

\section{INTRODUCTION}

$\mathbf{V}$ OLTAGE and current sensors are necessary components for the control, protection and power quality monitoring of electric power systems. There is a need in medium and high voltage networks, in particular in smart grids, for real-time sensors. In this paper a novel real-time Optical Voltage Sensor (OVS) for power network energy quality monitoring is demonstrated.

Manuscript received February 15, 2018; revised April 26, 2018; accepted May 21, 2018. Date of publication May 25, 2018; date of current version June 19, 2018. This work was supported by CAPES and National Council for Scientific and Technological Development, process number 420673/2016-4Brazil. (Corresponding author: Michael J. Connelly.)

F. C. Pereira, R. T. Higuti, and C. Kitano are with the Department of Electrical Engineering, Sao Paulo State University, Ilha Solteira 15385-000, Brazil (e-mail: fernandocp.eng@gmail.com; tokio@dee.feis.unesp.br; kitano@ dee.feis.unesp.br).

J. H. Galeti is with the Federal Institute of Mato Grosso do Sul, Tres Lagoas 79641-162, Brazil (e-mail: jhgaleti@gmail.com).

M. J. Connelly is with the Optical Communications Research Group, Department of Electronic and Computer Engineering, University of Limerick, Limerick V94 T9PX, Ireland (e-mail: michael.connelly@ul.ie).

Color versions of one or more of the figures in this paper are available online at http://ieeexplore.ieee.org.

Digital Object Identifier 10.1109/JLT.2018.2840706
In recent decades, considerable research efforts have been devoted to OVSs, and different physical principles and techniques have been investigated: piezoelectric effect with Bragg gratings [1], electro-optic effect in bulk devices [2], electro-optic effect in integrated optics [3], among others. This work focuses on OVSs based on the electro-optic effect in bulk crystals, because they constitute the most consolidated technology today. In this case, due to the anisotropic materials used, the voltage to be measured, $V_{s}(t)$, changes the polarization state of the light propagating through the crystal [4].

Electro-optical crystal-based OVSs, using bismuth germanate $\left(\mathrm{Bi}_{4} \mathrm{Ge}_{3} \mathrm{O}_{12}\right.$ or $\left.\mathrm{BGO}\right)$ or lithium niobate $\left(\mathrm{LiNbO}_{3}\right)$, for example, have been applied regularly in high-voltage systems due to their wide bandwidths, fast responses and small losses for optical transmission. The electric field applied to the crystals usually causes very small relative variations in the induced refractive indexes. However, such small variations are high enough for measuring the voltages of interest by using currently available electronic instrumentation. On the other hand, as the polarimetric technique is sensitive to very weak stimuli, in practice, the sensor output suffers from signal fading, particularly if the effects of natural birefringence of the electro-optical crystal are present.

In practical operations, the electro-optical crystal used as a sensor element in the OVS interacts not only with the electric field, but also with temperature and stress fields [5]. Because of this, bulk optic setups containing lenses, polarizing filters, waveplates, etc., make OVSs overly sensitive to temperature variations, mechanical shock and vibration, resulting in potentially unstable systems. Therefore, the detected signal may significantly fluctuate over time, due to these environmental agents. Thus, the challenge is to design an innovative OVS capable of meeting appropriate standards of accuracy (IEC, IEEE/ANSI) in the presence of undesirable external perturbations.

At the operational wavelength $\lambda=632.8 \mathrm{~nm}$, the electro-optic coefficient of $\mathrm{BGO}\left(\mathrm{r}_{41}\right)$ is smaller than those for $\mathrm{LiNbO}_{3}\left(\mathrm{r}_{33}\right.$, $r_{13}, r_{22}$ ) [4]. Therefore, the size of an OVS using BGO must be relatively larger in order to obtain a measurable phase shift and a good SNR [6]; in turn, it is possible to significantly reduce the size of the $\mathrm{LiNbO}_{3}$ Pockels cells. Consequently, when a $\mathrm{LiNbO}_{3}$ crystal is inserted between parallel electrodes, its small dimensions allow the effect of stray lines produced by the electric field near the electrode edges to be minimized, thereby, improving dynamic range, sensitivity and robustness of the sensor. Another problem is that BGO exhibits the magneto-optical effect 
(or Faraday effect), which can induce an undesirable angular variation of the optical field, generating errors in the electrooptical phase shifts [7]. The advantages mentioned above, together with the fact that $\mathrm{LiNbO}_{3}$ does not exhibit magnetooptical effect, justify its use for OVSs.

When the $\mathrm{LiNbO}_{3}$ Pockels cell exhibits natural birefringence, the OVS becomes highly sensitive to variations in ambient temperature. In order to solve this drawback, a passive compensation arrangement, called double-crystal configuration, consisting of two identical cascaded cells with a $\lambda / 2$ waveplate sandwiched between them was proposed [8].

As reported in the literature, even if the Pockels cell topology is such that the natural birefringence component is not present, crystal growth constraints and variations in environmental conditions can also lead to fading. This corresponds to the so-called "linear birefringence" [5], whose classical case is the birefringence induced by thermal gradients. In order to solve this problem, Pan et al. [9] proposed the use of an OVS based on a dual-crystal structure, consisting of two $\mathrm{LiNbO}_{3}$ crystals, identical in dimensions and axes orientation, but only one of the crystals (the sensor) was exposed to the electric field to be measured. The other element was a dummy crystal used for temperature compensation. The structure was completed by interleaving the cascade connection of the two crystals with a $\lambda / 2$ waveplate, such that the linearly polarized light vibrating along the $\mathrm{X}$-axis (Y-axis) in the compensation crystal vibrates along the Y-axis (X-axis) on the sensor crystal.

In addition to dual-crystal configurations, Li et al. [10] proposed a structure that was also double-pass, that is, the light that went through the cascaded system was reflected by a mirror film at the end of the last cell. In that system, the linear birefringence error in the sensor crystal is greatly reduced by the compensating crystal. However, the experimental setup is complex, involving additional elements such as an integrated optical phase modulator, an optical circulator, a Faraday rotator and a feedback system between the photodiode and modulator.

As with the OVSs described above, where waveplates were employed, the elimination of any such devices is desirable for simplifying the system, improving temperature stability and achieving insensitivity to light wavelength. Moreover, since such waveplates are separated from the sensor crystal, angular misalignments between their axes are usually unavoidable and lead to additional measurement errors [11]. To solve this problem, Li and Zeng [12] presented a classic BGO crystal-based arrangement, without the extra $\lambda / 4$ waveplate: the crystal was cut in such a manner that, from two internal total reflections, a relative phase shift of $\pi / 2$ between the $p$ and $s$ polarizing components (generated by the Fresnel reflection coefficients) was produced.

Most electro-optic OVSs used today are based on modification of basic configurations such as those described above. These configurations try to solve the signal-fading problem by adding more optical elements leading to increasingly complex and expensive setups.

An important observation is that there is a great similarity between the input-output characteristic of an OVS based on an optical-intensity electro-optic modulator composed of

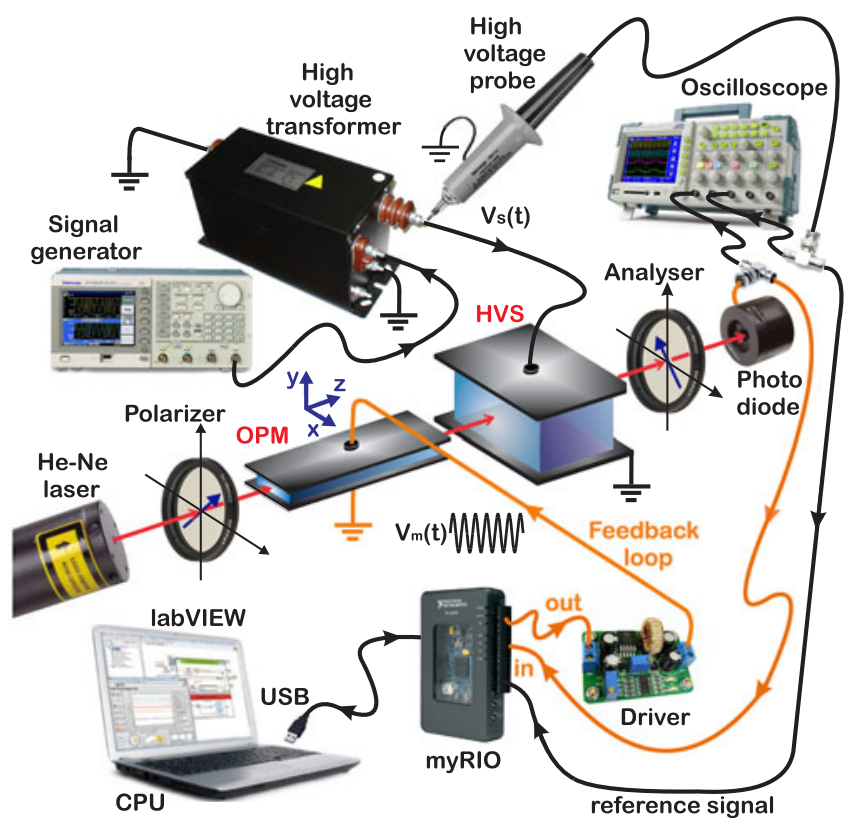

Fig. 1. OVS and feedback control loop experimental setup.

Pockels cell [4] and that of a two-beam interferometer, such as the Michelson or Mach-Zehnder [13]. This is because the arrangement of the intensity electro-optical modulator can be interpreted as a "polarimetric interferometer": the interference between the two propagation eigenmodes (constituting an elliptically polarized ray) after passing through an analyzer (polarizer at the system output), occurs. Consequently, most of the methods proposed to detect phase shift in a two-beam interferometer can be applied to OVSs.

Recently, a new Synthetic-Heterodyne (SH) method, employing the Hilbert transform and gain control feedback, was applied to a polarimetric-based OVS and used to measure high-voltage signals [14]. In this paper we describe an enhanced algorithm for optical phase demodulation, and a PI (Proportional-Integral) feedback control method. The optical hardware is kept simple, by using a polarimetric interferometer arrangement. It is constituted by the series association of a High-Voltage Sensor (HVS) Pockels cell with an Optical Phase Modulator (OPM) inserted between two crossed polarizers. No Hilbert transform or SH method is necessary, and the OPM signal does not need to be a sinusoid but can be a variety of periodic signals, thereby simplifying the detection method. In contrast to [14], data acquisition and signal processing for optical phase demodulation and control system operation are implemented in real-time. Frequency response linearity and response to waveforms with high harmonic content (up to the 25th component) and to voltage transients can be accurately obtained.

\section{PRoposed Method}

A schematic diagram of the proposed system is shown in Fig. 1, illustrating the $\mathrm{x}, \mathrm{y}$ and $\mathrm{z}$ coordinates corresponding to the reference axes. In the text, the axes of the $\mathrm{LiNbO}_{3}$ crystals will be denoted by $\mathrm{X}, \mathrm{Y}$ and $\mathrm{Z}$, where $\mathrm{Z}$ is the optical axis. The optical source is a $20 \mathrm{~mW}, 632.8 \mathrm{~nm} \mathrm{He}-\mathrm{Ne}$ laser. The laser beam 
is polarized at an angle of $45^{\circ}$ to the $y$-axis, in order to couple equal amplitudes of lightwave electric field to the orthogonal ordinary and extraordinary modes of the electro-optic crystals. These two rays propagate through the OPM, which is cascaded with HVS and are analyzed by a polarizer at an angle of $-45^{\circ}$ to the y-axis. The resulting light beam is detected by an amplified PIN photodiode (Thorlabs, PDA 55) and the resulting signal is digitized and processed in real-time using a reconfigurable $\mathrm{I} / \mathrm{O}$ device (National Instruments myRIO). A feedback adjustable amplitude sinusoidal waveform $V_{m}(t)$ is applied to the OPM by using an analog output from the myRIO.

The OPM is a bulk $\mathrm{LiNbO}_{3}$ Pockels cell having dimensions of $10,1.1$ and $50 \mathrm{~mm}$ in the $\mathrm{x}=\mathrm{Y}, \mathrm{y}=\mathrm{Z}$ and $\mathrm{z}=\mathrm{X}$ directions and optical propagation in the $\mathrm{z}=\mathrm{X}$ direction. The electric field corresponding to $V_{m}(t)$ is applied parallel to the crystal Z-axis. The total phase shift between the ordinary and extraordinary waves propagating though the OPM is sum of an electro-optic phase shift $\Phi(t)$, induced by $V_{m}(t)$, and a quasi-static phase shift $\Phi_{0}(t)$, caused by the natural birefringence of the crystal [4]. According to [8], $\Phi_{0}(t)$ varies slowly in time in a random way, and this variation is up to five orders of magnitude greater than that caused by $\Phi(t)$. Following [4], the OPM theoretical half-wave voltage can be calculated as $V_{\pi m}=64.92 \mathrm{~V}$. This relatively small value of $V_{\pi m}$ provides the OPM cell with the capability to realize electronic adjustments to compensate the fading effect at the HVS cell output due to environmental drifts. The driver, shown in Fig. 1, interfaces the photodiode output to the myRIO and also enables the myRIO output to drive the OPM.

The HVS is a bulk $\mathrm{LiNbO}_{3}$ Pockels cell with dimensions of $20,9.92$ and $10.26 \mathrm{~mm}$ along $\mathrm{x}=\mathrm{X}, \mathrm{y}=\mathrm{Y}$ and $\mathrm{z}=\mathrm{Z}$ directions, respectively, and optical propagation in the $\mathrm{z}=\mathrm{Z}$ direction. The voltage to be measured, $V_{s}(t)$, corresponds to an electric field applied parallel to the crystal Y-axis. In this configuration, there is no natural birefringence present in the total phase shift between the eigenmodes. But, because of the linear birefringence effect [5], the total phase shift is equal to the sum of $\phi(t)$, the phase shift induced by $V_{s}(t)$, and $\varphi(t)$, a slowly time varying phase shift caused by environmental drifts. Although the variation in $\varphi(t)$ is smaller than the variation in $\Phi_{0}(t)$, it seriously affects the accuracy in the measurement of $V_{s}(t)$. Following [4], the HVS half-wave voltage theoretical value is $V_{\pi s}=3.77 \mathrm{kV}$. As shown in Fig. 1, the high-voltage $V_{s}(t)$ applied to the HVS cell is produced at the secondary winding of a dry $\mathrm{HV}$ transformer, whose primary winding is fed by a digital signal generator (followed by a power amplifier, not shown in Fig. 1). $V_{s}(t)$ is monitored by an oscilloscope with a calibrated $\mathrm{HV}$ probe.

\section{A. OVS Analysis}

The optical system shown in Fig. 1 is very similar to the intensity modulator discussed in [4], when the $\lambda / 4$ waveplate used is substituted by the OPM. Thus, by analogy, the photodetected signal is given by:

$$
v_{p d}(t)=A\left\{1-V \cos \left[\Phi(t)+\Phi_{0}(t)+\phi(t)+\varphi(t)\right]\right\}
$$

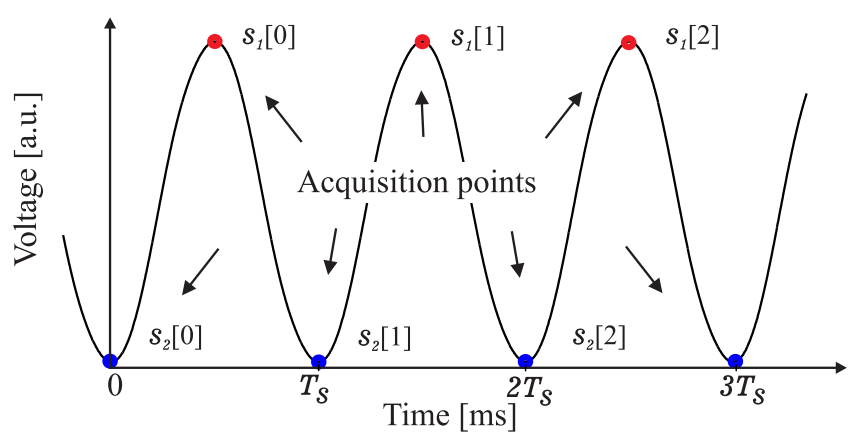

Fig. 2. Maxima and minima points of $V_{m}(t)$ waveform acquired at $2 f_{0}$ sample rate synchronously.

where $A$ is a constant voltage and $V$ is the fringe visibility $(0<V<1)$, inserted to take into account for any misalignment between laser polarization and/or light propagation direction and the crystal axes.

Considering a sinusoidal $V_{m}(t)=V_{m} \cos \left(2 \pi f_{0} t\right)$ having frequency $f_{0}$, with an amplitude $V_{m}=V_{\pi m} / 4$, the phase-shift induced by the OPM is [4]:

$$
\Phi(t)=\pi \frac{V_{m}(t)}{V_{\pi m}}=\frac{\pi}{4} \cos \left(2 \pi f_{0} t\right) .
$$

The phase shift induced by the HVS is

$$
\phi(t)=\pi \frac{V_{s}(t)}{V_{\pi s}} .
$$

By substituting (2) in (1) and defining

$$
\theta(t)=\Phi_{0}+\phi(t)+\varphi(t)+\frac{\pi}{4},
$$

and subtracting the $A$ from $v_{p d}(t)$, the following signal results:

$$
\begin{aligned}
v(t) & =-A V \cos \left[\Phi(t)+\theta(t)-\frac{\pi}{4}\right] \\
& =-A V \cos \left[\frac{\pi}{4} \cos \left(2 \pi f_{0} t\right)+\theta(t)-\frac{\pi}{4}\right] .
\end{aligned}
$$

When $\Phi(t)$ is a sinusoidal waveform like (2), (5) can also be written as a Fourier series in terms of Bessel functions of the first kind and order $i, J_{i}(C)$, for a specified $C$-factor. This was done in [15], when the SH method with gain feedback control was used to detect $\theta(t)$, by using its first and second harmonic components, $J_{1}(C)$ and $J_{2}(C)$. In the present work, however, another approach is proposed, in which the Fourier series representation is not necessary.

In our method, $v(t)$ must be acquired synchronously with the periodic OPM voltage $V_{m}(t)$ using a sample rate $f_{s}=2 f_{0}$, where $f_{0}=12.5 \mathrm{kHz}$. A typical $V_{m}(t)$ applied to the OPM and the corresponding acquired signal $v(t)$ are shown in Figs. 2 and 3, respectively. In Fig. 3, the sampling points were joined by straight-line segments to provide a better visualization of the two envelopes, which are in quadrature to each other. There are two samples per period of $V_{m}(t)$.

An important characteristic of the method is that the acquisition of $v(t)$ is synchronized with $V_{m}(t)$, in such a way that the samples are taken at the instants where $V_{m}(t)$ reaches its minima and maxima. At these instants, from the form of (5), 


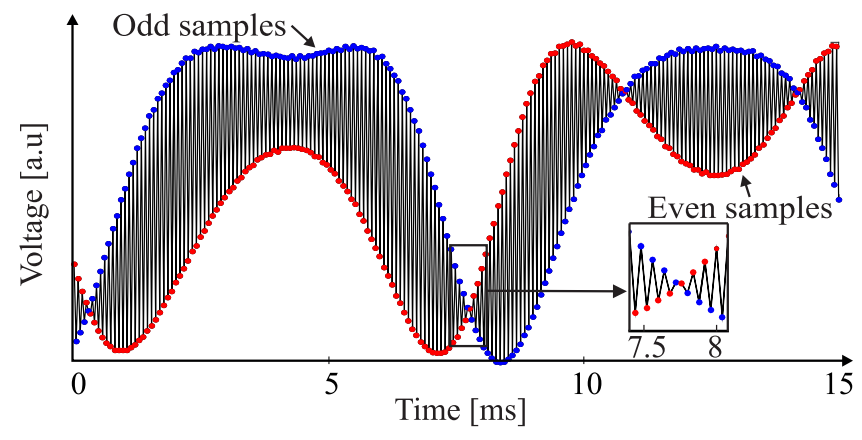

Fig. 3. Typical acquired $v(t)$ divided into odd and even samples. The inset shows a detailed view of $v(t)$ near crossing region.

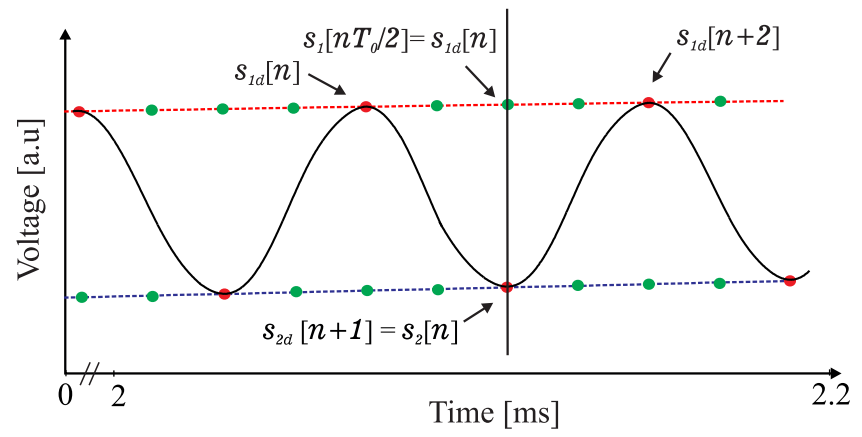

Fig. 4. Schematic representation of $s_{1 d}$ and $s_{2 d}$ interpolation and delaying $s_{1 d}[n]$ by two samples.

$\Phi(t)$ assumes values of $\pi / 4$ and $-\pi / 4$, respectively, and the sampled signal $v[n]=v\left(n T_{s}\right), n=0,1,2, \ldots$, results in:

$$
v[n]=v\left(n T_{s}\right)= \begin{cases}-A V \cos \theta\left(n T_{s}\right), & n \text { even } \\ -A V \sin \theta\left(n T_{s}\right), & n \text { odd }\end{cases}
$$

The decimated sequences $s_{1 d}[n]=v[2 n]$ (even samples, red) and $s_{2 d}[n]=v[2 n+1]$ (odd samples, blue) are obtained, and represent samples of the envelopes of the function $v(t)$, each with a sampling period $T_{0}=1 / f_{0}$. It is important to note that $s_{1 d}[n]$ and $s_{2 d}[n]$ represent samples at different instants of time: for example, $s_{1 d}[0]$ is the sample of $v(t)$ at $t=0$ while $s_{2 d}[0]$ is $v(t)$ sampled at $T_{s}=T_{0} / 2$. Consequently, for correct signal manipulation, interpolation and shifting are required, as described below.

$s_{1 d}[n]$ and $s_{2 d}[n]$ are interpolated by spline method with an even factor $L$ (e.g., 4, when 3 samples are created between two existing samples), resulting in envelope signals with an equivalent sample period of $T_{0} / L$, without a significant load on myRIO. These signals are called $s_{1}[n]$ and $s_{2}[n]$. In order to represent the signals with the correct relative delay $T_{0} / 2$, a relative shift of $L / 2$ samples must be applied. In the case of $L=4$, this can be obtained by delaying $s_{1}[n]$ by one sample and advancing $s_{2}[n]$ by one sample, or just delaying $s_{1}[n]$ by two samples, as shown in Fig. 4.

Consequently, the following envelope signals result, after interpolation and appropriate time-shifting (the same signals are used for simplicity):

$$
\begin{aligned}
& s_{1}[n]=-\left.A V \cos \theta(t)\right|_{t=n T_{0} / L}=\left.s_{1}(t)\right|_{t=n T_{0} / L} \\
& s_{2}[n]=-\left.A V \sin \theta(t)\right|_{t=n T_{0} / L}=\left.s_{2}(t)\right|_{t=n T_{0} / L}
\end{aligned}
$$

representing the quadrature envelopes of $v(t)$, sampled at a rate equal to $L f_{0}$.

This constitutes the essence of the method: by switching $\Phi(t)$ in (5), between $-\pi / 4 \mathrm{rad}$ and $+\pi / 4 \mathrm{rad}$ at the sampling instants, quadrature terms can be generated consecutively by interlaced acquisition, and thus, both envelopes can be obtained from just one photodiode. These two envelopes allow the phase shift $\theta(t)$ to be determined by using any classical quadrature detection technique, such as the arctan method. However, in practice this would demand some procedure to correct the quadrature condition [16] as well as a phase unwrapping algorithm [17]. These methods require many time-consuming procedures, which are difficult to implement in real-time. Nevertheless, a different, innovative and efficient method will be described as follows.

Now manipulating the equivalent continuous time-domain signals, it can be shown that

$$
s_{3}(t)=s_{1}(t) \dot{s}_{2}(t)-s_{2}(t) \dot{s}_{1}(t)=(A V)^{2} \dot{\theta}
$$

where the dot represents differentiation. $\dot{\theta}(t)$ can be obtained independent of the factor $A V$ by using the relation

$$
S_{v}(t)=\frac{s_{3}(t)}{s_{1}^{2}(t)+s_{2}^{2}(t)}=\dot{\theta}(t) .
$$

The proposed method is self-consistent, once $S_{v}$ is independent of spurious variations of the product $A V$, thereby improving measurement stability and accuracy. Assuming that $\dot{\phi}(t) \gg \dot{\varphi}(t)$ and $\dot{\phi}(t) \gg \dot{\Phi}_{0}$ (typically, temperature induced variations in $\varphi(t)$ and $\Phi_{0}(t)$ have frequencies less than a few $\mathrm{Hz}$ ), which is a reasonable assumption in practice, highpass filtering $S_{v}(t)$ where $\theta(t)$ is given by (4), leads to the filtered signal $S_{v f}(t)=\dot{\theta}(t)=\dot{\phi}(t)$. $V_{s}(t)$ can be recovered by time integration and substituting the result in (3) to obtain

$$
V_{s}(t)=\frac{V_{\pi s}}{\pi} \int S_{v f}(t) d t .
$$

So, $V_{s}(t)$ is measured independently of its waveform and of random phase shifts occurring in $\Phi_{0}(t)$ or $\varphi(t)$.

The method has a number of advantages. For instance, in the SH demodulation scheme used in our previous work [14], the carrier wave $V_{m}(t)$ must be a sinusoidal in order to avoid second harmonic distortion. This presented method does not require a pure sinusoidal $V_{m}(t)$ as a carrier wave, a very distorted sinusoidal by superior harmonics signals can be used, as detailed ahead in the experimental results at Fig. 8. Another advantage is that the quadrature terms with $\sin \theta(t)$ and $\cos \theta(t)$ could be processed to obtain a signal proportional to $\theta(t)$, as proposed in [15] by using SH demodulation, where the Fourrier components at $f_{0}$ and $2 f_{0}$ were used in order to achieve the maximum detectable measurand signal bandwidth, of $f_{0} / 2$. However, in that case the minimum received signal bandwidth, which allowed successful measurand signal demodulation was $2.5 f_{0}$, and according to the Nyquist sampling theorem it would require a 
minimum sampling rate of $5 f_{0}$, i.e., 10 times the bandwidth of the measured signal. Our new method requires only four times the sampling rate, by using a half of the sampling rate for the required samples $s_{1}[n]$ and $s_{2}[n]$.

However, the method depends on the temperature sensitivity of the half-wave voltages $V_{\pi s}$ and $V_{\pi m}$, and the accuracy of the synchronous acquisition process. These limitations can be greatly reduced by the use of a control loop described in the following section.

\section{Phase FeEdBack Control}

Among the few studies that have already realized the potential of modern control in the theoretical and experimental analysis of OVSs, we can cite the following: [11], [14], [18], [19]. Essentially, the purpose of the OVS described in Fig. 1 is to measure the $V_{s}(t)$, according to (11). However, $V_{\pi s}$ can vary with temperature, causing inaccuracies in this measurement procedure. Also, the method described in Section II assumes that (2) is satisfied, i.e., $V_{m}=V_{\pi m} / 4$. However, in practice, $V_{\pi m}$ also varies with temperature. Finally, the adequate operation of the optical sensors depends on the synchronous acquisition process, which must be precisely tuned to sample $v(t)$ at the instants of maxima and minima of $V_{m}(t)$. A feedback control system can be used to compensate for variations in $V_{\pi s}$ and $V_{\pi m}$, and also compensate for timing inaccuracies in the synchronous acquisition.

\section{A. Measurement of the Constant Amplitude A}

The IEC 61869-3 standard, concerning instrument transformer HV (including OVSs) power quality measurements, imposes a well defined accuracy (class dependent) for a voltage between $80 \%$ and $120 \%$ of its nominal value $V_{n}$ [20]. The signal processing algorithm and control loop shown in Fig. 5 is designed to have the operating around the desired nominal value of $V_{s}(t)$. As discussed, $V_{\pi m}$ can vary due to temperature drifts, causing variations in the desired value of $V_{m}$ of $V_{\pi m} / 4$. Something similar can occur with the constant voltage $A$, which must be subtracted from (1) in order to generate (5): as the value of $A$ depends on the stability of the light source, photodetector responsivity and the gain of acquisition system, among others, its value can also vary undesirably in time. Because of this, measurement of $A$ and the proper adjustment of $V_{m}$ must be carried out in real-time. After this procedure, the algorithm described in Fig. 5 is executed, and the instantaneous value of $V_{s}(t)$ is obtained. The process is repeated for the next acquisition.

According to Appendix, in order to simplify the determination of $A$ it is appropriate that the OVS operates under multifrinje regime, and so, the HVS Pockels cell must be adequatily chosen. Proper operation of the OVS, under the worst case (the limit between single fringe and multifringe regime) for controlling $V_{s}(t)$, in real-time and accurately, can be achieved by selecting a HVS cell whose $V_{\pi s}$ is $\leq 0.8 V_{n}$, leading to a phase shift $\phi(t)$ whose peak value is higher than $\pi \mathrm{rad}$, as can be seen from (3). This enables measurement of $A$ (see Appendix) and control of the amplitude of $V_{m}(t)$ such that it attains a desired amplitude of $V_{\pi m} / 4$. If the selected HVS works well for $0.8 V_{n}$, it will also works when $V_{s}(t)>120 \%$ of $V_{n}$.

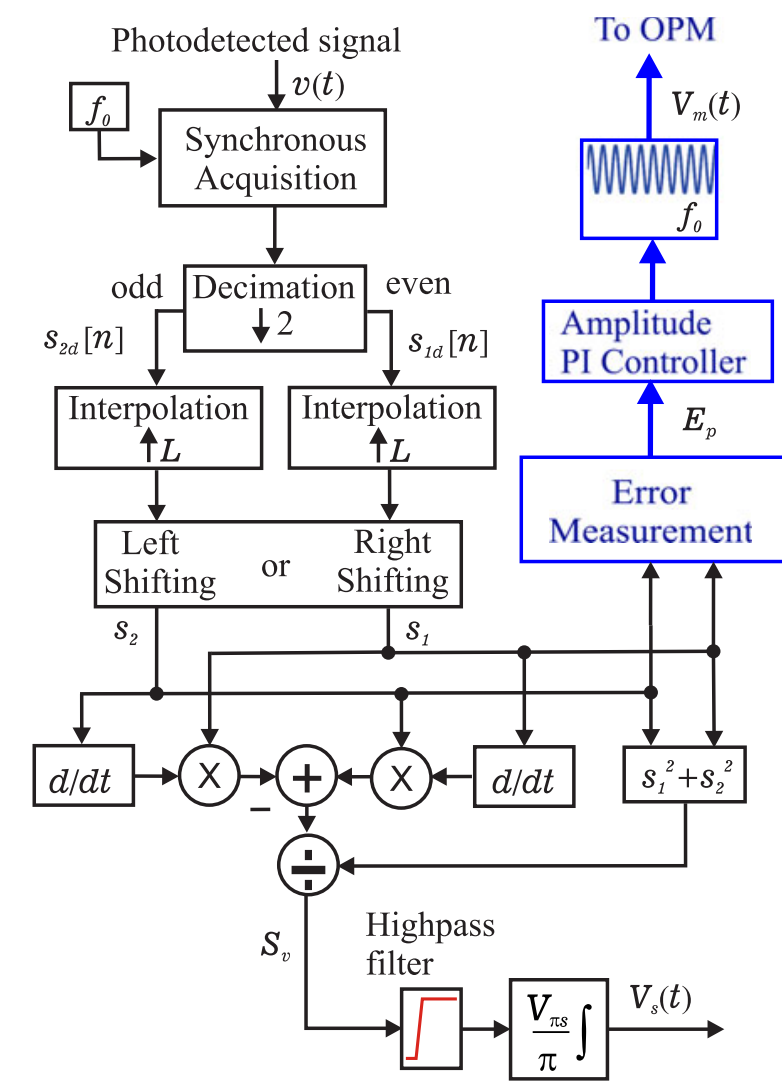

Fig. 5. Schematic diagram of the proposed method and feedback control system.

\section{B. Error Signal Generation}

In practice, it can be shown that the ideal expressions (7) and (8) can be substituted by:

$$
\begin{aligned}
& s_{1}[n]=-A V \cos \left[\theta\left[n T_{s}\right]\right] \\
& s_{2}[n]=-A V \sin \left[\theta\left[n T_{s}\right]+\delta\right],
\end{aligned}
$$

where $\delta$ is the phase shift error induced by $V_{m}(t)$ drift from the ideal value $V_{\pi m} / 4$, due to acquisition timing error and $V_{\pi m}$ fluctuations. The control system target is hold $\delta=0$. From (2) the phase induced by $V_{m}(t)$ drift at (12) and (13) is rated as $V_{\pi m} / \pi$. Considering phase shift error $\delta$ only at (13) (without prejudice to the generality), the drifted $V_{m}(t)$ can be written as:

$$
V_{m}^{\prime}=V_{m}+\Delta V_{m}=\frac{V_{\pi m}}{4}+\frac{V_{\pi m}}{2 \pi} \delta,
$$

The effect of $\delta$ also can be seen in the Lissajous figure of Fig. 6 (for $\delta=\pi / 4$ ). In this example, the sum of areas in quadrants 1 and 3 (odd quadrants) is larger than the sum of areas in quadrants 2 and 4 (even quadrants). When $\delta=0$, the ellipse becomes a circle, all four quadrants have equal areas, and (12) and (13) return to the ideal signals (7) and (8), respectively.

Measurement $\delta$ in real-time is not simple, so an equivalent error signal, $E_{p}$, that varies linearly with delta around the desired OVS operating point, is proposed. Instead of directly working with quadrant areas, it is more convenient to use the ellipse coordinates $\left(s_{1}[n], s_{2}[n]\right)$ to calculate a suitable form of $E_{p}$. When $s_{1}$ and $s_{2}$ have the same sign, an estimate of the 


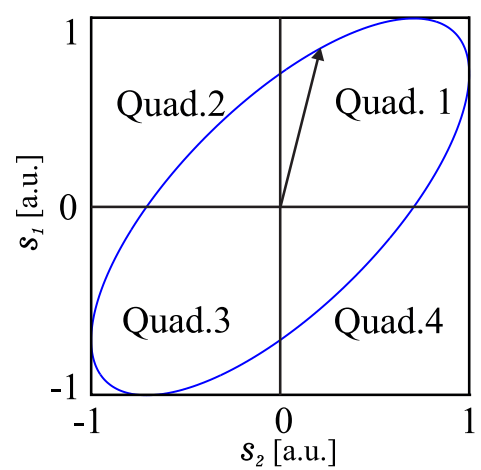

Fig. 6. Experimental Lissajous figure derived $s_{1}$ and $s_{2}$ when $\delta=\pi / 4$.

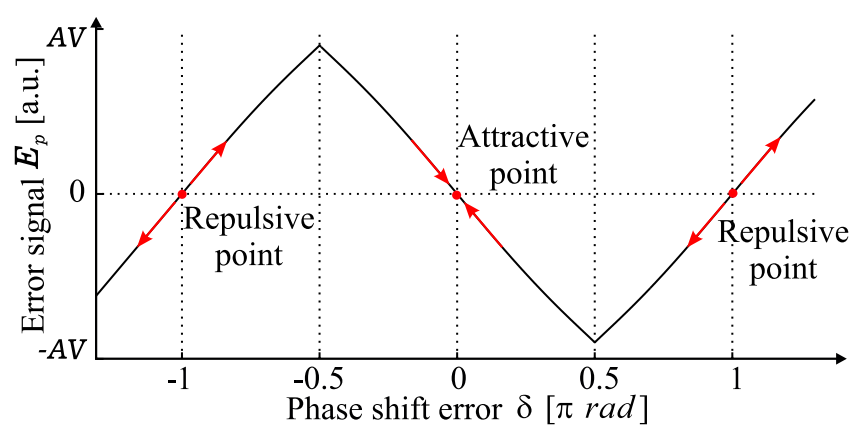

Fig. 7. Relationship between $E_{p}$ and $\delta$ error signals.

semi-major axis is given by

$$
R_{13}=\frac{1}{p} \sum_{n=1}^{p} \sqrt{s_{1}^{2}[n]+s_{2}^{2}[n]},
$$

where $p$ is the number of samples of $s_{1}$ and $s_{2}$ having the same sign. When the signs of $s_{1}$ and $s_{2}$ are different, a estimate of the semi-minor axis is given by:

$$
R_{24}=\frac{1}{q} \sum_{n=1}^{q} \sqrt{s_{1}^{2}[n]+s_{2}^{2}[n]}
$$

where $q$ is the number of samples of $s_{1}$ and $s_{2}$ with different signs. The error signal is defined as:

$$
E_{p}=R_{13}-R_{24} \text {. }
$$

The integers $p$ and $q$ can be different in each sampling. There are no specific values for $p$ or $q$ because $R_{13}$ and $R_{24}$ are averages. In practice, the $E_{p}$ calculation starts not matching the theoretical curve (Fig. 7) when $p+q$ is less than 10 samples (for constant sampling rate) per cycle (at $60 \mathrm{~Hz}$ ); however, aiming to discriminate $6 \mathrm{kHz}$ (for example) we must use at least 200 samples per cycle. At this work, 10 cycles of $60 \mathrm{~Hz}$ was sampled at $12.5 \mathrm{kHz}$, such that $p+q=2083$.

Next, the control loop shown in Fig. 1 (and also in Fig. 5) is used to nullify $\delta$ by controlling $V_{m}^{\prime}$ in (14), aiming $E_{p}=0$. Substituting (12) and (13) into (15) and (16), and hereafter, both results in (17), Fig. 7 is obtained, which shows the approximately linear dependence of $E_{p}$ on $\delta$ around the desired operation point, where $\delta=0$.

The $E_{p}$ is convenient error signal to be used as an input of a simple P or PI controller aiming keep the phase error $\delta=0$,

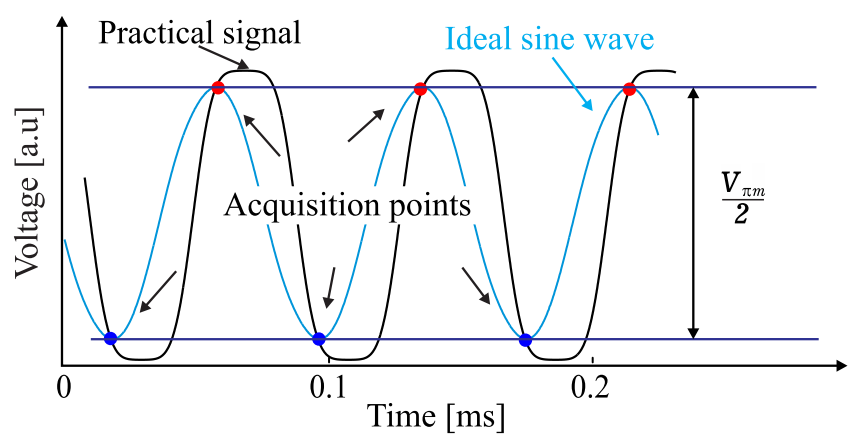

Fig. 8. Synchronization carried out with distorted $V_{m}(t)$ signal.

when the signals (12) and (13) would be in phase quadrature condition. Whenever $\delta<0$, i.e., between the minimum practical $\delta=-\pi / 2$ value (when $V_{m}^{\prime}=0$ in (14)) and $\delta=0^{-}$(Fig. 7), the $E_{p}>0$, driving the controller to increase $V_{m}^{\prime}$. However when $\delta$ is in between 0 and $\pi$, the $E_{p}<0$, driving the controller to decrease $V_{m}^{\prime}$, tending to keep $\delta=0$ with $V_{m}^{\prime}$ around $V_{\pi m} / 4$, at the so named attractive point in Fig. 7. Likewise, it may be concluded that points $\delta=+\pi$ and $\delta=-\pi$ are repulsive points.

By utilizing $E_{p}$ and a labVIEW standard controller block (basic PID - Proportional-Integral-Derivative controller) the feedback system (the PI block shown in Fig. 5) was designed to stabilize the OVS in real-time. The PI was tuned using the heuristic method of Ziegler-Nichols and the derivative part was disabled.

\section{Practical Synchronous Acquisition System}

A fundamental characteristic of the feedback controller is related to the synchronous acquisition. As $V_{\pi m}=64.92 \mathrm{~V}$, in practice, it is possible that the voltage $V_{m}(t)$ needs to assume tens of volts in order to stabilize the OVS. Although a sinewave is the simplest waveform to be generated by signal generators, it can be distorted when it needs power amplification (or increased amplitude via transformers) or buffering. However, this is not important once our OVS is capable to operate with a large number of periodic $V_{m}(t)$ signals, as the distorted square waveform shown in Fig. 8. Thus, given a fixed sampling frequency $f_{0}$, whenever a non-zero error signal is sent to the PI controller, it analyzes the $E_{p}$ value and adjusts the amplitude of $V_{m}(t)$ accordingly, such that the voltage difference between two successive samples be equal to $V_{\pi m} / 2$. In fact, since the value of $V_{\pi m}$ itself can vary with temperature, the amplitude of $V_{m}(t)$ is set to an amount that is independent of the actual value of $V_{\pi m}$. This amplitude is adjusted by the control system to keep $\delta=0$ without the need of a pure sinusoidal modulation, which is something intolerable in SH methods such as [14], [15].

\section{EXPERIMENTAL RESULTS}

An important performance factor in OVS systems, rarely reported in the literature, is the overall bandwidth, considering the OVS itself, data acquisition and digital signal processing of the photodetector signal. One of the objectives of this work is to measure this parameter, and also, to establish a compromise between the frequency bandwidth and the sampling rate in order to 


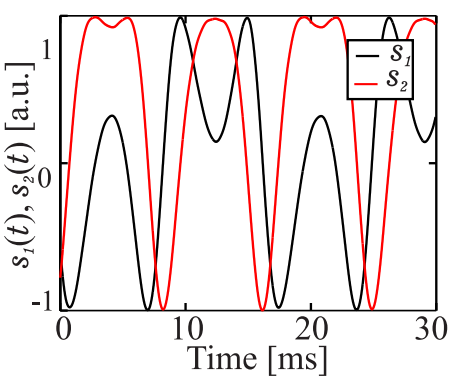

(a)

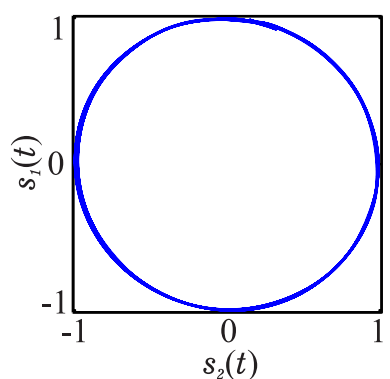

(b)
Fig. 9. Experimental $s_{1}(t)$ and $s_{2}(t)$ signals when $E_{p}=0$. (a) In time domine. (b) As Lissajous figure.

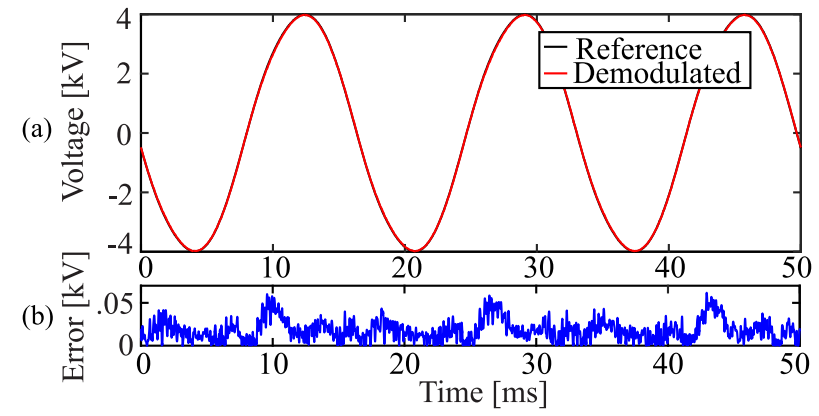

Fig. 10. (a) Reference and demodulated $V_{s}(t)$ and (b) absolute difference for a sinusoidal signal applied to the OVS.

result in a cost effective system. Another concern is to evaluate whether the demodulation method of the photodetected signal is suitable for real-time operation.

The practical values of OPM and HVS half-wave voltages $\left(V_{\pi m}\right.$ and $V_{\pi m}$, respectively) were measured by using the Signal Coincidence Method (SCM) described in ISO 16063-41 [21], [22], resulting in $V_{\pi m}=62.3 \mathrm{~V}$ and $V_{\pi s}=4.03 \mathrm{kV}$.

The OVS system was designed to enable operation around $5 \mathrm{kV}( \pm 20 \%)$. Typical $s_{1}(t)$ and $s_{2}(t)$ signals that occur when $E_{p}=0$ are shown in Fig. 9(a). When $E_{p}=0, s_{1}(t)$ and $s_{2}(t)$ are in quadrature and have equal peak values. The respective Lissajous figure (normalized by the peak values) is shown in Fig 9(b).

The main interest for developing the OVS is the measurement of power line harmonic distortion, which is an issue where a wide frequency bandwidth is important. The voltages detected with our method were compared to that measured by a high voltage probe (Tektronix P6015A, $28 \mathrm{kV}, 75 \mathrm{MHz}$ ) connected to the HVS.

\section{A. OVS Linearity Analysis}

The OVS linearity was tested by applying different voltage levels to the HVS cell and measuring the values of demodulated voltage. Typical demodulated and reference signals are shown in Fig. 10(a), corresponding to the response for a $60 \mathrm{~Hz}, 4 \mathrm{kV}$ amplitude sinusoidal voltage. Absolute difference (in volts) is shown in Fig. 10(b), revealing that there was a very good agreement between them, with a deviation of less than $50 \mathrm{~V}$.

Figure 11 shows the demodulated voltage as a function of the reference voltage, when sinusoidal signals at $60 \mathrm{~Hz}$ frequency

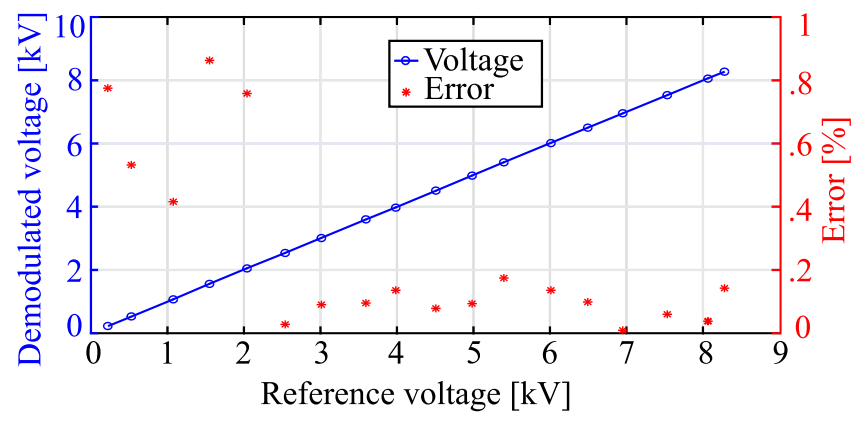

Fig. 11. Comparison between demodulated and reference voltages and relative error.

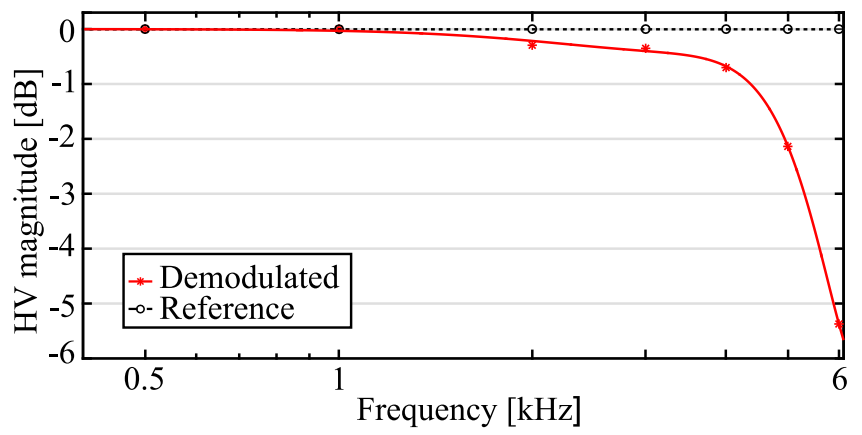

Fig. 12. Frequency response of the OVS and the reference probe.

and amplitude between 200 and $8200 \mathrm{~V}$ (peak) are applied to the sensor. Therefore, the linearity of the sensor is excellent, and in the range between 2.5 and $8 \mathrm{kV}$, the control system is in normal operation, and the maximum reading errors are less than $0.2 \%$, i.e., the difference between reference and demodulation voltage divided by reference voltage.

\section{B. OVS Frequency Response}

In the frequency response shown in Fig. 12, the ratios between the OVS output and input high voltage amplitudes are plotted as a function of frequency, between $500 \mathrm{~Hz}$ and $6 \mathrm{kHz}$. The flat frequency response of the $\mathrm{HV}$ probe is also shown. The detection bandwidth $(-3 \mathrm{~dB})$ is approximately $5 \mathrm{kHz}$. In the following, it is demonstrated that the sensor bandwidth is sufficient.

\section{Total Power Line Harmonic Distortion}

The Total power line Harmonic Distortion (THD) is evaluated according to [20]:

$$
\mathrm{THD}=\frac{\sqrt{\sum_{i=2}^{25} h_{i}^{2}}}{h_{1}},
$$

where $h_{i}$ is amplitude of the $i$-th harmonic of the measured $V_{s}(t)$.

In order to measure THD, a highly distorted $4 \mathrm{kV}$ signal was applied to the HVS. The demodulated and reference signals and difference between them are shown in Fig. 13. The maximum difference is approximately $50 \mathrm{~V}$.

The spectrum of the demodulated signal shown in Fig. 13 is shown in Fig. 14, along with the reference signal spectrum. Odd 
(a)

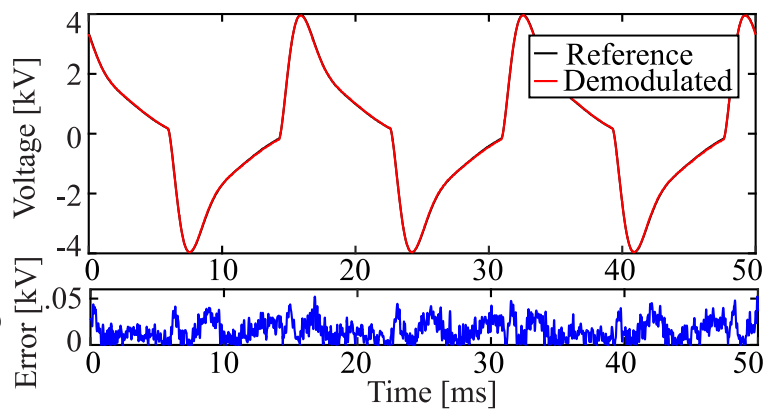

Fig. 13. (a) Demodulated and reference signals and (b) absolute error for a highly distorted waveform applied to HVS.

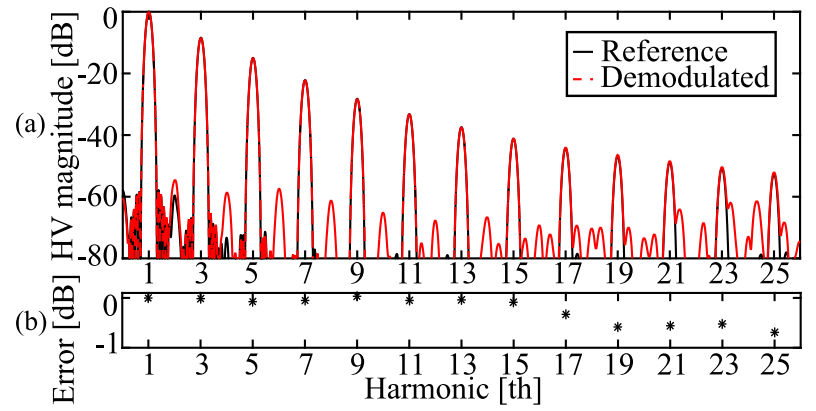

Fig. 14. (a) Spectra of the demodulated and reference signals shown in Fig. 13 , and (b) the respective error. The fundamental corresponds to a frequency of $60 \mathrm{~Hz}$.

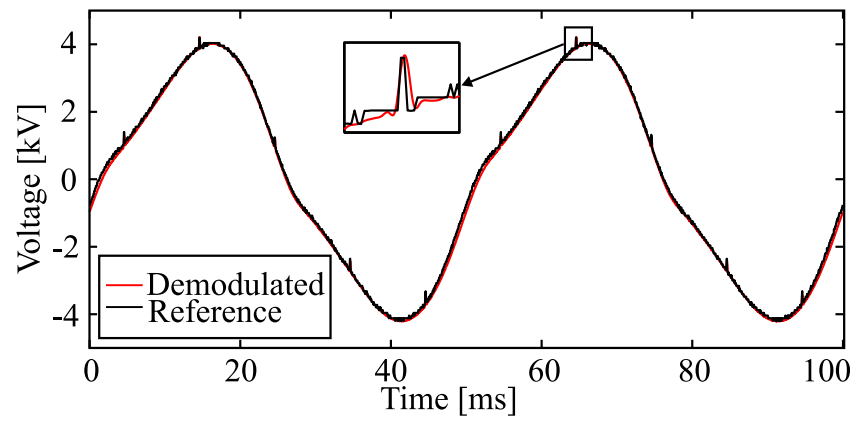

Fig. 15. OVS and reference responses in the presence of transient disturbances.

harmonics are predominant and there is good agreement between both spectra up to the 25 th harmonic, with the maximum difference of less than $-1 \mathrm{~dB}$.

The THD measured with the reference probe was $42.87 \%$, while the value obtained with the OVS was $42.99 \%$, showing that in the frequency range up to $1.5 \mathrm{kHz}$, the optical sensor has performs almost as well as the reference probe.

\section{Response to Transient Disturbances}

Power quality has become a significant matter for power utilities, end users and electric and electronic equipment manufacturers due to the increase in non-linear loads. They are difficult to detect because they have a short duration, usually much shorter than the fundamental period [23].

In order to simulate transient disturbances, a $320 \mathrm{~V}$ amplitude pulse train $(100 \mathrm{~Hz}$ frequency and $40 \mu$ s pulse width) was added to a distorted sinusoidal voltage ( $20 \mathrm{~Hz}$ and $4.08 \mathrm{kV}$ amplitude). In Fig. 15 it can be seen that the OVS is capable of accurate measurement of transient.

\section{CONCLUSION}

A cost-effective polarimetric OVS using feedback control was proposed, by using synchronous acquisition of the photo detected output signal in order to obtain two signals in phase quadrature, which are used for demodulation of the induced phase shift. The novel method has several advantages: regarding [14] and [15], no spectral decomposition of the photo detected signal through FFT is required, as well as no Hilbert transform (both requiring high processing resources). Consequently, the new method is faster, requiring lower sampling rates when compared to the SH method, allowing the use of off-the shelf signal acquisition and processing solutions and real-time operation. The method is self-consistent, not depending on the product $A V$ present in (5), as well as the random phase shifts $\Phi_{0}(t)$ and $\varphi(t)$ that are present in (1). The feedback control system is constituted by a simple PI controller, actuated by an error signal whose reference is a circular Lissajous figure. Measurements were performed with sinusoidal signals at $60 \mathrm{~Hz}$, for voltages between 0 and $8.2 \mathrm{kV}$ peak, and errors less than $0.2 \%$, thus satisfying the IEC 61869-3 standard. The OVS frequency bandwidth, taking into account the myRIO signal processing unit, was greater than that obtained by [14]. High-voltages having high harmonic content and signals with transient disturbances were measured with good accuracy, when compared to masurements obtained with a reference HV probe.

\section{APPENDIX \\ MEASUREMENT OF THE CONSTANT $A$}

Simple DC coupling is not suitable to remove the constant $A$ from (1) because the cosine term in (1) has a significant non-zero DC component.

Hence, it becomes important to measure the value of $A$ before executing any further measurements. For many applications this can be achieved, as a first step, by gradually increasing the voltage applied to the HVS, $V_{s}(t)$, until $\phi(t)$ is $>\pi$. Under this condition, the $v_{p d}(t)$ will present many cycles per period of $V_{s}(t)$, all of them having the same peak-to-peak value. $A$ can be calculated as $A=\left(\left(v_{p d}\right) \max +\left(v_{p d}\right) \min \right) / 2$, where $\left(v_{p d}\right) \max$ and $\left(v_{p d}\right)$ min are the maximum and the minimum values of the $v_{p d}(t)$ respectively. The next step is calculate $v(t)=v_{p d}-A$, as in (5), which can be straightforwardly applied and the control system can use real-time values, since the measured voltage with the proposed OVS is around nominal ( $5 \mathrm{kV} \pm 20 \%)$.

\section{REFERENCES}

[1] A. Dante, R. M. Bacurau, A. W. Spengler, E. C. Ferreira, and J. A. S. Dias, "A temperature-independent interrogation technique for FBG sensors using monolithic multilayer piezoelectric actuators," IEEE Trans. Instrum. Meas., vol. 65, no. 11, pp. 2476-2484, Nov. 2016.

[2] J. C. J. de Almeida and J. C. Santos, "Coherent demodulation of the output signal of optical voltage transformer," IEEE Latin Amer. Trans., vol. 3, no. 5 , pp. 47-52, Dec. 2005. 
[3] P. P. Chavez, N. A. F. Jaeger, F. Rahmatian, and C. P. Yakymyshyn, "Integrated-optic voltage transducer for high-voltage applications," Proc. SPIE, vol. 4087, pp. 1229-1237, Dec. 2000.

[4] A. Yariv and P. Yeh, Optical Waves in Crystals: Propagation and Control of Laser Radiation. New York, NY, USA: Wiley, Jan. 1983.

[5] X. Xiao, Y. Xu, and Z. Dong, "Thermodynamic modeling and analysis of an optical electric-field sensor," Sensors, vol. 15, no. 4, pp. 7125-7135, Mar. 2015.

[6] Q. Yang, S. Sun, R. Han, W. Sima, and T. Liu, "Intense transient electric field sensor based on the electro-optic effect of $\mathrm{LiNbO}_{3}$," $A I P$ Adv., vol. 5, no. 10, 2015, Art. no. 107130.

[7] A. Kumada and K. Hidaka, "Directly high-voltage measuring system based on Pockels effect," IEEE Trans. Power Del., vol. 28, no. 3, pp. 13061313, Jul. 2013

[8] W. Sima, T. Liu, Q. Yang, R. Han, and S. Sun, "Temperature characteristics of Pockels electro-optic voltage sensor with double crystal compensation," AIP Adv., vol. 6, no. 5, May 2016, Art. no. 055109.

[9] F. Pan, X. Xiao, Y. Xu, and S. Ren, "Optical AC voltage sensor based on two $\mathrm{Bi}_{4} \mathrm{Ge}_{3} \mathrm{O}_{12}$ crystals," IEEE Trans. Instrum. Meas., vol. 61, no. 4, pp. 1125-1129, Apr. 2012.

[10] L. Li, W. Zhang, H. Li, and R. Pan, "Linear birefringence-free optical voltage sensor based on dual-crystal structure," Appl. Opt., vol. 52, no. 36, pp. 8706-8713, Dec. 2013.

[11] W. Deng, H. Li, C. Zhang, and P. Wang, "Optimization of detection accuracy of closed-loop optical voltage sensors based on Pockels effect," Sensors, vol. 17, no. 8, Jul. 2017, Art. no. 1723.

[12] C. Li and R. Zeng, "Optical voltage sensor using single Fresnel rhomb $\mathrm{Bi}_{4} \mathrm{Ge}_{3} \mathrm{O}_{12}$ crystal," IEEE Sensors J., vol. 14, no. 1, pp. 79-84, Jan. 2014.

[13] E. Udd and W. B. S. Jr., Fiber Optic Sensors: An Introduction for Engineers and Scientists, 2nd ed. New York, NY, USA: Wiley, Oct. 2011.

[14] J. H. Galeti, R. T. Higuti, C. Kitano, and M. J. Connelly, "Polarimetric optical high-voltage sensor using synthetic-heterodyne demodulation and Hilbert transform with gain control feedback," IEEE J. Sel. Topics Quantum Electron., vol. 23, no. 2, pp. 417-423, Mar. 2017.

[15] J. H. Galeti, C. Kitano, and M. J. Connelly, "Improved syntheticheterodyne Michelson interferometer vibrometer using phase and gain control feedback," Appl. Opt., vol. 54, no. 35, pp. 10418-10424, Dec. 2015.

[16] P. L. M. Heydemann, "Determination and correction of quadrature fringe measurement errors in interferometers," Appl. Optics, vol. 20, no. 19, pp. 3382-3384, Oct. 1981 .

[17] T. Usuda, M. Dobosz, and T. Kurosawa, "Evaluation method for frequency characteristics of linear actuators in the sub- $m u$ m stroke range using a modified michelson-type interferometer," Nanotechnology, vol. 9, no. 2, Jun. 1998, Art. no. 77.

[18] L. Liu, H. Li, Z. Fu, and L. Feng, "Analysis on the optimization of high-frequency performance for optical voltage sensors based on pockels effect," IEEE Sensors J., vol. 17, no. 15, pp. 4826-4833, Aug. 2017.

[19] H. Li, L. Cui, X. Wang, Z. Lin, and C. Zhang, "Analysis and design of loop gains to optimize the dynamic performance of optical voltage sensor based on Pockels effect," J. Lightwave Technol., vol. 33, no. 14, pp. 3108-3115, Jul. 2015.

[20] Instrument Transformers - Part 3: Additional Requirements for Inductive Voltage Transformers, IEC/TR-61869-3, IEC, Jul. 2011.

[21] Methods for the Calibration of Vibration and Shock Transducers - Part 41: Calibration of Laser Vibrometers, ISO 16063-41, Aug. 2011.

[22] Methods for the Calibration of Vibration and Shock Transducers - Part 11: Primary Vibration Calibration by Laser Interferometry, ISO 1606311, Dec. 1999.

[23] J. Barros and R. I. Diego, "A review of measurement and analysis of electric power quality on shipboard power system networks," Renew. Sustain. Energy Rev., vol. 62, pp. 665-672, Sep. 2016.
Fernando da Cruz Pereira received the B.Sc. degree in telecommunication engineering from the Centro Universitário Católico Salesiano Auxilium, Araçatuba, Brazil, in 2010, and the M.Sc. degree in electrical engineering from Universidade Estadual Paulista, São Paulo, Brazil, in 2013, where he is currently working toward the Ph.D. degree. His current research interests include interferometry, automation, nanodisplacement measurements, piezoelectric actuators, and optical high-voltage sensors.

José Henrique Galeti (M'12) received the B.Sc. degree in electrical engineering from the Escola de Engenharia Mauá, São Paulo, Brazil, in 1988, the M.Sc. degree in electrical engineering from Universidade Estadual Paulista, São Paulo, Brazil, in 2012, and the Ph.D. degree in 2016. He was with Hewlett-Packard Brazil, São Paulo, Brazil, and from 1988 to 2009, he was with ETS-Electronic Test Systems, São Paulo, Brazil, where he was involved in test and measurement industrial applications. His current research interests include interferometry, automation, nanodisplacement measurements, piezoelectric actuators, and optical high-voltage sensors.

Ricardo Tokio Higuti (M'00) received the B.S. degree in electrical engineering and the M.S. and Dr. Eng. degrees in mechanical engineering from the Escola Politécnica da Universidade de São Paul, São Paulo, Brazil, in 1991, 1994, and 2001, respectively. Since 1995, he has been with the Department of Electrical Engineering, Universidade Estadual Paulista, São Paulo. His current research interests include signal processing, the development of ultrasonic sensors for liquids, and nondestructive testing.

Michael J. Connelly (S'89-M'92-SM'14) received the B.E. and Ph.D. degrees in electronic engineering from the National University of Ireland, Dublin, Ireland, in 1987 and 1992, respectively. He is an Associate Professor in electronic engineering and the Director of the Optical Communications Research Group, University of Limerick, Limerick, Ireland. His current research interests include coherent optical communication systems, semiconductor optical amplifiers, optical coherence tomography, and optical interferometry.

Cláudio Kitano received the Diploma degree in electrical engineering from the Universidade Estadual Paulista (UNESP), São Paulo, Brazil, the Master's degree in electronics engineering from the Technological Institute of Aeronautics (ITA), São José dos Campos, Brazil, and the D.Sc. degree in microwave and optoelectronics from ITA, in 2001. Since 1987, he has been with the Department of Electrical Engineering, UNESP. His current research interests include optical interferometry, optical fiber sensors, integrated optics, photothermal science, elastic guided waves, acoustic optic, and optical high-voltage sensors. 